\title{
Mehr als eine Frage von Konzept und Geld
}

\section{Nachhaltige Entwicklung ist eine Herausforderung für alle Bereiche der Gesell- schaft. Die Wissenschaft kann einerseits die langfristigen Folgen nicht-nachhal- tigen Handelns aufzeigen und andererseits Zukunftsvisionen und konkrete Ge- staltungsoptionen entwickeln. Damit die Universitäten diesem Auftrag gerecht werden, müssen sie über Fachgrenzen hinaus denken und stärker in öffentliche Debatten eingreifen. Das Forschungsprogramm „Forschung für die Nachhaltig- keit" kann diese Entwicklungen unterstützen.}

$\mathrm{D}$ Von Angelika Zabrnt ie Frage, nach der Rolle der Wissenschaft für die Umsetzung einer Nachhaltigen Entwicklung, möchte ich gerne mit Hilfe eines Beispiels beantworten. Die Studie „Zukunftsfähiges Deutschland" war die erste umfangreiche und konkrete Studie zu den Anforderungen einer nachhaltigen Entwicklung in Deutschland. Sie war in der Öffentlichkeit ein großer Erfolg, doch vor allem war sie forschungspolitisch ein gelungenes Nachhaltigkeitsexperiment.

Der Ausgangspunkt für die Entstehung der Studie war die Gegenkonferenz der Nichtregierungsorganisationen zum G7-Gipfel in München 1992, auf der Manus van Brakel über die Studie „Sustainable Netherlands" berichtete. Der Versuch, Nachhaltigkeit konkret für die praktische Nachhaltigkeitspolitik in den Niederlanden zu übersetzen, hatte große öffentliche Resonanz gefunden. Der Bund für Umwelt und Naturschutz Deutschland (BUND) griff den Impuls auf, ließ die niederländische Studie übersetzen und löste damit eine mit großem öffentlichen Interesse begleitete Diskussion aus.

\section{Studie für Deutschland}

Es entstand die Idee, eine gleiche Studie für Deutschland zu entwickeln. Sie sollte zeigen, was die Bekenntnisse von Rio für ein Industrieland wie die Bundesrepublik in der Nachhaltigkeitspolitik konkret bedeuten würden. Gemeinsam mit dem entwicklungspolitischen Partner Misereor wurde als wissenschaftlicher Auftragnehmer das Wuppertal-Institut ausgewählt. Den Ausschlag dafür gab dessen Bereitschaft und Fähigkeit zu interdisziplinärem und transdisziplinärem Arbeiten mit Praxispartnern sowie die Bereitschaft zu einem etwas ungewöhnlichen Forschungsablauf: Während der Erstellung waren immer wieder Beratungsschritte mit den Praxispartnern vorgesehen. Zur Halbzeit fand eine wissenschaftliche Fachdiskussion in größerem Kreis statt, die auch zu einer deutlichen Korrektur des Projekts führte. Gleichzeitig mit der wissenschaftlichen Erarbeitung wurde von BUND und Misereor ein umfangreiches Kommunikationskonzept erarbeitet.

In diesem Projekt wurden inzwischen gängige Anforderungen an Nachhaltigkeitsforschung umgesetzt Die internationale Anbindung, die Interdisziplinarität, die Transdisziplinarität, die Sondierungsphase, die Ausgestaltung als lernendes Projekt und die anschließende Kommunikationsphase.

Nachhaltigkeit ist per se ein integratives Konzept, das die drei Bereiche Ökonomie, Soziales und Ökologie miteinander vernetzt. Die komplexen Probleme, vor denen die Gesellschaft steht, erfordern eine konstruktive Zusammenarbeit der Vertreter verschiedener Disziplinen. Dabei versteht sich von selbst dass die Schwerpunkte je nach Fachbereich unterschiedlich gesetzt werden müssen, um dem je eigenen fachlichen Anspruch gerecht zu werden.

\begin{tabular}{c}
\hline Nachbaltige \\
Firmengründung \\
ist das Thema \\
des Informationsdienstes \\
Ökologisches Wirtschaften \\
$2 / 05$ \\
Wenn Sie potenzielle Beiträge haben, \\
wenden Sie sich bitte \\
an die Redaktion.
\end{tabular}

\section{- Vielfältige Anforderungen}

Die Anforderungen bei der Auseinandersetzung mit dem Thema Nachhaltigkeit sind vielfältig. Um das Thema adäquat zu bearbeiten, bedarf es der Auseinandersetzung mit dem so genannten Systemwissen, das heißt Wissen sowohl um Wirkungszusammenhänge, Funktionsweisen von Strukturen und Prozesse als auch darüber hinausgehende Wissensbestände um kulturelle Hintergründe. Systemwissen beinhaltet ausserdem tradiertes Wissen über den Umgang mit Natur, Erfahrungen aus der Alltagsorganisation, die Berücksichtigung verschiedener Sichtweisen. Alle Wissensbereiche stehen auf der Basis einer ethischen Orientierung zum Verhältnis von Mensch und Natur.

Auf dem Weg zur Forschung für Nachhaltigkeit gilt es eine Reihe von Widerständen zu überwinden. Der erste ist die „Bildungs-Provinzialität“, in der die universitäre Lehre allzu gerne verharrt. Die große Gefahr ist, dass die Universität ihrem eigenen interdisziplinären Forschungspotenzial hinterherhinkt. In der Zersplitterung der Disziplinen und ihrer weiteren Ausdifferenzierung muss sich die Universität den Vorwurf gefallen lassen, immer noch viel zu sehr das tief gehende Spezialwissen zu honorieren. Wer den Blick über den Tellerrand der eigenen Disziplin wagt, wird selten belohnt. Nimmt die Universität die Herausforderung der Interdisziplinarität an, sichert sie ihre eigene Zukunftsfähigkeit.

Denn international wettbewerbsfähige Forschung erfordert besonders bei zukunftsträchtigen Wissensgebieten, die starke disziplinäre Orientierung aufzugeben. Wie fundamental hier die Widerstände sind, zeigt sich, wenn man Vertreter verschiedener Fachbereiche vor ein Problem stellt. Ihre Lösungen lassen an babylonisches Sprachgewirr erinnern, weil sich die Fachwissenschaftler trotz derselben Verhandlungssprache durch ihre eigenen Denkweisen fundamental unterscheiden. Die große Herausforderung auf dem Weg zur fruchtbaren Interdisziplinarität ist die Schulung des methodischen, reflexiven Bewusstseins.

Der zweite Widerstand sind Wissenschaftler, die mit ihrer Wissenschaft nicht normativ in den öffentlichen Diskurs einzugreifen wollen. Doch die Frage, ob sich Wissenschaft hier einmischen darf, ist vollkommen unangebracht. Die Fragen sollte vielmehr lauten: Kann es die Wissenschaft verantworten, sich bei normativen Fragen wie der Verringerung des Fleischkonsums, der zunehmenden Mobilität, des Flächenverbrauchs durch ständig steigende Wohnansprüche nicht einzumischen? 


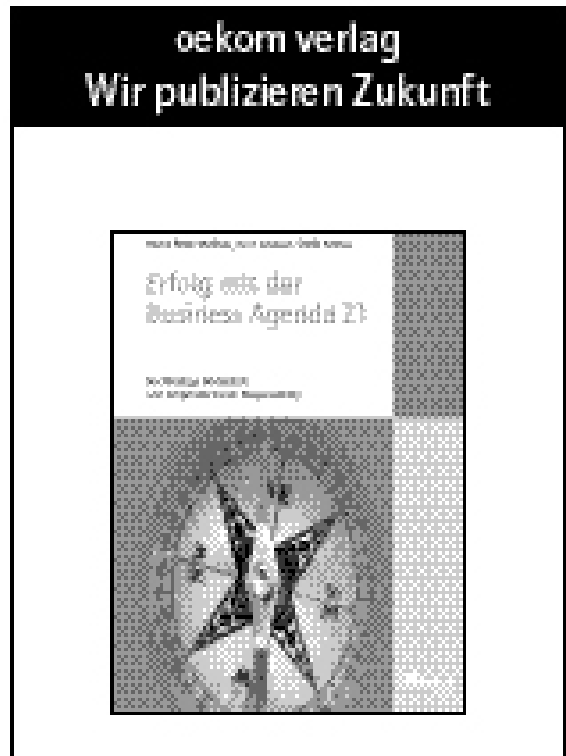

Heirz Peter Wallner, Kurt Schauer, Dodo Kresse

Erfolg mit der Business Agenda 21

\section{Nach haltige Wirtschaft und} Corporate Social Responsibility

Auch för Entscheider [-innen] aus der Wirtschaft ist es immer wichtiger zu wissen wie man auf Markten mit Erfalg agie en kann, in denen der Aspekt Nachhaltigkeit eine entschei dende Rolle spielt

De Autoren (innen) beeiten in ihrem Buch anschaulich dax aktuelle Wissen uber nachhaltiges Wirtschaften vor dem Hintergnund never Ziele und Bedu ifnisse der Ceselkshaft auf. Mit den 7 Maknahmen der Business Agent da 21 entwickein sie einen Wegaeiser for innovative Manager(inn)en der Unterneh men hilft, eine erfolgreiche und nachhaltige Ceschaftsstrategie zu finden.

oeloom verisg; Monchen 2004;

280 Seiter 26,50 E (rnd. Versand. hosten]; ISBN 7936581-770

Erhaltich be i

wnivorlagsorvice GntH S CO. KG Ausibforurg oobomverlay. 日6950 lardaben an lech

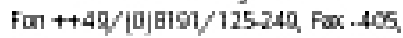
EMal f. mongiew niwerlagse wice de
Sind Fragen der Einstellung, sind Lebensbilder der wissenschaftlichen Bearbeitung anders als deskriptiv zugänglich? Sind Werte Privatsache oder Politik? Ist der Wissenschaft derRückzug in rein reaktives Verhalten erlaubt nach dem Motto: Liebe Menschen, ändert Ihr erst mal eure Werthaltungen, dann liefern wir euch die Handlungskonzepte? Wissenschaft kann sich hier nicht heraus halten. Im Gegenteil, sie hat im Kontext praktischer Nachhaltigkeitspolitik ein erweitertes Aufgabenspektrum. Sie muss

- die Konsequenzen des gegenwärtigen nichtnachhaltigen Handelns aufzeigen,

- selbst Zukunftsentwürfe für nachhaltige Entwicklungen formulieren und die Entwicklung gesellschaftlicher Zukunftsentwürfe durch die Gesellschaft unterstützen und Methoden hierzu entwickeln,

- Instrumente und Maßnahmen für ein Umsteuern in Richtung Nachhaltigkeit entwickeln,

- Bedingungen gesellschaftlicher Veränderungsprozesse analysieren, um die Grenzen bestehender Strukturen zu verschieben; denn die Verlierer bei Veränderungen sind in der Regel gut identifizierbar und gut organisiert, die potenziellen Gewinner oft nicht erkennbar und demzufolge auch nicht organisiert und als Lobby wirksam,

- die Behäbigkeit, wenn nicht gar Widerspenstigkeit von Institutionen und Bürokratien gegenüber Veränderung analysieren und Bedingungen für Kursänderung und Innovation erarbeiten,

- analysieren, wie beim Individuum die Macht der Gewohnheit angegangen werden kann, um neue Verhaltensweisen zu erproben und dauerhaft zu übernehmen.

Das Problem mag für die Wissenschaft darin liegen, dass sich Wissenschaftler viel zu selten in einer entschiedenen und offensiven Weise an den öffentlichen Kontroversen beteiligen und die aufklärerische Kraft der Wissenschaft beweisen. Doch ist es an der Zeit, dass sich die Wissenschaft in öffentliche Debatten einbringt. Entscheidend für den Erfolg der gestalterischen Einmischung ist jedoch, ob Wissenschaftler und Wissenschaftlerinnen durch die Originalität, die Differenziertheit und die Genauigkeit der Analysen, also mit unverkennbarer fachlicher Kompetenz zu überzeugen und zu beeindrucken vermögen. Der Erfolg des Themas Nachhaltigkeit hängt zu großen Teilen davon ab, inwiefern es gelingt, die Zukunftsvision zu konkretisieren und greifbar zu machen. Die große Chance im Diskurs mit der Gesellschaft ist die gestalterische Kraft, mit der sich die Wissenschaft daran beteiligen kann.

\section{Dialoge für die Zukunft}

Das zeigt auch der Dialog Nachhaltigkeit und Gesellschaft des Nachhaltigkeitsrates. Er fragte unter anderem, welche Beiträge von der Institution Wissenschaft zu erwarten seien und welche Kompetenzen man der Wissenschaft zumessen könne. Die Frage nach dem ,was wollen wir?"“ und ,wo wollen wir hin" wird immer drängender. Wir befinden uns in einer Krise der modernen Zivilisation, die ethische Dimensionen hat. Dieser Tatsache kann sich auch die Wissenschaft nicht entziehen. Die logische Konsequenz aus der Krise ist, dass sich auch die Anforderungen an Wissenschaft verändern. Change Management ist nicht nur ein Thema für Unternehmensberater. Change Management ist ein wissenschaftliches Aufgabenfeld für die gesamte Gesellschaft, wenn das neue Ziel Nachhaltigkeit nicht nur proklamativ sondern handlungsleitend sein soll.

\section{- Neve Impulse}

Ein positives Zeichen für das Umdenken an den Universitäten ist die verstärkte Gründung von Wissenstransferzentren, die den Universitäten Ansehen und Einfluss verschaffen. Im Moment droht jedoch aufgrund der aktuellen finanziellen Lage und der immer größeren Abhängigkeit der Universitäten von Drittmittelfinanzierung die Gefahr, sich möglicherweise vor den Karren der Politik oder der Wirtschaft spannen lassen zu müssen. Das Forschungsrahmenkonzept „Forschung für Nachhaltigkeit“ (FONA) setzt neue Impulse. Die Nachhaltigkeitsforschung wird als eine begrüßenswerte Initiative zur Vitalisierung der Wissenschaftslandschaft in Deutschland betrachtet. Es besteht Hoffnung, dass sie mit ihren Forschungsergebnissen einen Beitrag zur Nachhaltigkeit leistet, indem sie die Adressaten aus Wirtschaft sowie Aus- und Weiterbildung erreicht. Sollte dies der Fall sein, wäre das Programm ein guter Ansatz für das innovative Miteinander von Forschung, Wirtschaft und Gesellschaft.

\section{Die Autorin}

Dr. Angelika Zahrnt ist Vorsitzende des Bundes für Umwelt und Naturschutz Deutschland (BUND) und Mitglied des Nachhaltigkeitsrates der Bundesregierung.

Kontakt: Nachhaltigkeitsrat, Reichspietschufer 50, 10785 Berlin. E-Mail: angelika.zahrnt@bund.net 
(c) 20I0 Authors; licensee IÖW and oekom verlag. This is an article distributed under the terms of the Creative Commons Attribution Non-Commercial No Derivates License (http://creativecommons.org/licenses/by-nc-nd/3.o/), which permits unrestricted use, distribution, and reproduction in any medium, provided the original work is properly cited. 